\title{
Recommandations pour la prise en charge des prématurés à la limite de la viabilité (22-26 semaines de gestation)
}

Groupe de travail de la Société Suisse de Néonatologie*

\section{Résumé}

Le fotus ou nouveau-né à la limite de la viabilité (22-26 semaines de gestation) doit être pris en charge par une équipe périnatologique multidisciplinaire expérimentée. Deux facteurs influenceront tout particulièrement la prise en charge de chaque cas individuel: la précision limitée de la détermination de l'âge gestationnel et la variabilité biologique.

Les décisions à prendre sont complexes et lourdes en conséquences pour le long terme. Elles doivent être élaborées au cours d'un dialogue continu entre médecins, soignants et parents afin de prendre les mesures les plus adéquates dans le meilleur intérêt de l'enfant.

La connaissance des statistiques actuelles de mortalité et de morbidité selon l'âge de gestation et l'adoption des principes de base éthiques reconnus doivent constituer les fondements pour une prise en charge responsable et adéquate. La communication entre toutes les personnes amenées à prendre des décisions joue un rôle central.

Sur la base des données actuellement disponibles sur la mortalité et la morbidité à long terme, la prise en charge des prématurés d'un âge de gestation < 24 semaines devra en règle générale se limiter à des mesures palliatives.

Lors de prématurité $\geq 24$ semaines de gestation, la décision quant à la pertinence d'une prise en charge intensive incombe à une équipe de néonatologie expérimentée. L'option pour des soins intensifs «provisoires» (provisional intensive care) permet souvent une évaluation plus détaillée de l'état du prématuré, avec un certain recul afin de mieux pouvoir argumenter en faveur d'une poursuite ou de l'arrêt des mesures intensives.

Les mesures entreprises devront être continuées aussi longtemps que les évaluations rapprochées permettent un espoir justifié de survie du prématuré avec une qualité de vie acceptable et que les soins nécessaires prodigués sont raisonnables.
Si par contre l'équipe soignante et les parents doivent reconnaître que les soins prodigués engendrent des souffrances au delà de l'acceptable par rapport au résultat attendu, les mesures intensives perdent alors leur sens, et d'autres aspects de la prise en charge (par exemple l'utilisation d'opiacés pour soulager douleurs et souffrances) deviennent prioritaires (redirection of care). Si l'on renonce à des mesures intensives (primairement ou secondairement), tout devra alors être entrepris pour permettre à l'enfant un décès digne (comfort care). Un soutien des parents dans l'accompagnement de leur enfant dans la mort est indispensable.

\section{Introduction}

Ces recommandations concernent la prise en charge de prématurés avec un âge gestationnel entre 22 et 26 semaines. Elles s'adressent à l'intention des médecins, sages-femmes, infirmières, puéricultrices et membres d'autres groupes professionnels engagés dans la prise en charge d'enfants extrêmement prématurés (par simplification, la forme masculine sera utilisée lors de mention de personnes). Ces recommandations ont été élaborées par une commission d'experts mandatés par la Société Suisse de Néonatologie et elles ont été approuvées par la Société Suisse de Gynécologie et Obstétrique (SSGO), la Société Suisse de Pédiatrie (SSP) et la Société Suisse de Néonatologie (SSN).

La Commission Centrale d'Ethique (CCE) de l'Académie Suisse des Sciences Médicales (ASSM) supporte ces recommandations.

Ce document est en partie basé sur des recommandations européennes, américaines et canadiennes [1-4] ainsi que sur les directives médico-éthiques de l'Académie Suisse des Sciences Médicales [5, 6].
Neugeborenenintensivstation

CH-6000 Lucerne

Tél. 0412053285

e-mail: thomas.berger@ksl.ch 
Tableau 1

Comparaison entre différentes statistiques de mortalité: EPICure Study Group (Angleterre, données 1995) [8], El-Metwally et al. (Rhode Island, données 1993-1997) [10], Jacobs et al. (Toronto, données 1990-1994) [9], Swiss Minimal Neonatal Data Set (MNDS, données 1996 et 2000 ).

\begin{tabular}{|c|c|c|c|c|}
\hline Etude (année de publication) & $220 / 7-226 / 7$ SG & $230 / 7-236 / 7$ SG & $240 / 7-246 / 7$ SG & $250 / 7-256 / 7$ SG \\
\hline EPICure Study Group (2000) & $136 / 138(98 \%)$ & $216 / 241(90 \%)$ & $284 / 382(74 \%)$ & $241 / 424(57 \%)$ \\
\hline El-Metwally et al. (2000) & $21 / 22(95 \%)$ & $22 / 41(54 \%)$ & $25 / 61(41 \%)$ & $16 / 87(18 \%)$ \\
\hline Jacobs et al. (2000) & - & $44 / 56(79 \%)$ & $38 / 90(42 \%)$ & $52 / 138(38 \%)$ \\
\hline MNDS 1996 (non publiée) & - & - & $12 / 14(86 \%)$ & $28 / 54(52 \%)$ \\
\hline MNDS 2000 (non publiée) & - & $5 / 5(100 \%)$ & $23 / 28(82 \%)$ & $33 / 62(53 \%)$ \\
\hline
\end{tabular}

\section{Considérations préliminaires}

\subsection{Calcul de I'âge gestationnel et variabilité biologique}

Selon l'International Classification of Disease (10 ${ }^{\text {th }}$ revision), l'âge gestationnel est défini par l'âge post-menstruel et indiqué en semaines et jours. Selon cette définition, la période entre 25 semaines et 0 jour ( $250 / 7$ semaines de gestation) et 25 semaines et 6 jours ( $256 / 7$ semaines de gestation) par exemple, sera définie comme $25^{\mathrm{e}}$ semaine de gestation révolue (175-181 jours, completed weeks of gestation); le fœtus aura donc terminé sa $25^{\mathrm{e}}$ semaine et se trouve dans la $26^{\mathrm{e}}$ semaine de gestation.

Le calcul de l'âge gestationnel se base en règle générale sur des mesures ultrasonographiques de la longueur céphalo-caudale au début de la grossesse ( $8^{\mathrm{e}}$ à $12^{\mathrm{e}}$ semaine) et/ou sur les indications anamnestiques de la date des dernières règles. Il faudra aussi tenir compte de la précision méthodologique qui est de l'ordre de \pm 4 jours pour l'ultrason précoce [7] et de -6 à +14 jours pour les indications anamnestiques. Si aucune évaluation obstétricale précoce du terme n'est disponible, l'évaluation clinique postnatale de l'enfant permet parfois une révision de l'âge gestationnel. Les parents devraient être informés de cette imprécision avant l'accouchement afin d'éviter une désécurisation des parents.

La variabilité biologique peut aussi être à l'origine de différences de maturité considérables entre prématurés d'âge gestationnel identique. De telles différences de maturité pourront se refléter également sur les besoins thérapeutiques du nouveau-né ainsi que sur ses risques de mortalité et de morbidité.

\subsection{Epidémiologie: mortalité et morbidité actuelle}

Le développement continu de la néonatologie intensive n'a cessé d'abaisser les limites de la viabilité (limit of viability), à tel point qu'aujourd'hui une survie après 22 semaines de gestation révo- lue a été décrite [4, 8]. La comparaison entre les chiffres internationaux publiés [8-10] et les résultats du Swiss Minimal Neonatal Data Set (MNDS) montre des différences considérables de mortalité entre différents centres (tabl. 1). D'autre part, des études internationales récemment publiées prouvent clairement une augmentation du risque de handicap résiduel lorsque l'âge gestationnel diminue (fig. 1) [8], tout en démontrant de grandes différences interinstitutionnelles $[8,9]$.

L'équipe périnatale doit connaître les statistiques actuelles de mortalité et morbidité selon l'âge gestationnel. Ces données épidémiologiques doivent contenir les informations sur l'incidence et la gravité des handicaps à l'âge de 2 ans et plus. A côté des données nationales et internationales, les résultats locaux sur le devenir des prématurés à la limite de la viabilité sont d'une importance primordiale.

\subsection{Réflexions éthiques}

La mortalité et morbidité élevées (tabl. 1, fig. 1) soulèvent des questions graves et ont amené à une prise de conscience importante concernant les aspects éthiques de la prise en charge des prématurés à la limite de la viabilité.

Lors d'une prise en charge d'un fotus ou d'un prématuré à la limite de la viabilité, les parents et l'équipe soignante doivent prendre ensemble les décisions quant aux mesures les plus appropriées, et ce dans le meilleur intérêt pour cet enfant. Les quatre principes éthiques de Beauchamp et Childress [11], l'autonomie, l'innocuité (le principe de ne pas nuire), le bénéfice (le principe d'être bénéfique) et la justice serviront de guide aussi pour les prématurés. L'application de ces principes à la situation du prématuré à la limite de la viabilité est souvent difficile et conflictuelle [12].

2.3.1 Devoir médical quant au maintien de la vie Un premier point conflictuel consiste en l'influence potentielle d'une qualité de vie espérée sur les choix médicaux face au maintien de la vie. 


\section{Figure 1a}

Mortalité et morbidité neurologique de prématurés d'âge gestationnel entre 22 0/7 et 23 6/7 (1995). Le développement psychomoteur a été évalué à l'âge de 30 mois (médian). Rectangles gris foncés: dénominateur = enfants nés vivants (live born infants), rectangles gris clairs: dénominateur = enfants admis en néonatologie (admissions to NICU), rectangles blancs: dénominateur = enfants vivants à la sortie d’hôpital (survivors to discharge). Donnés selon EPICure Study Group, 2000.
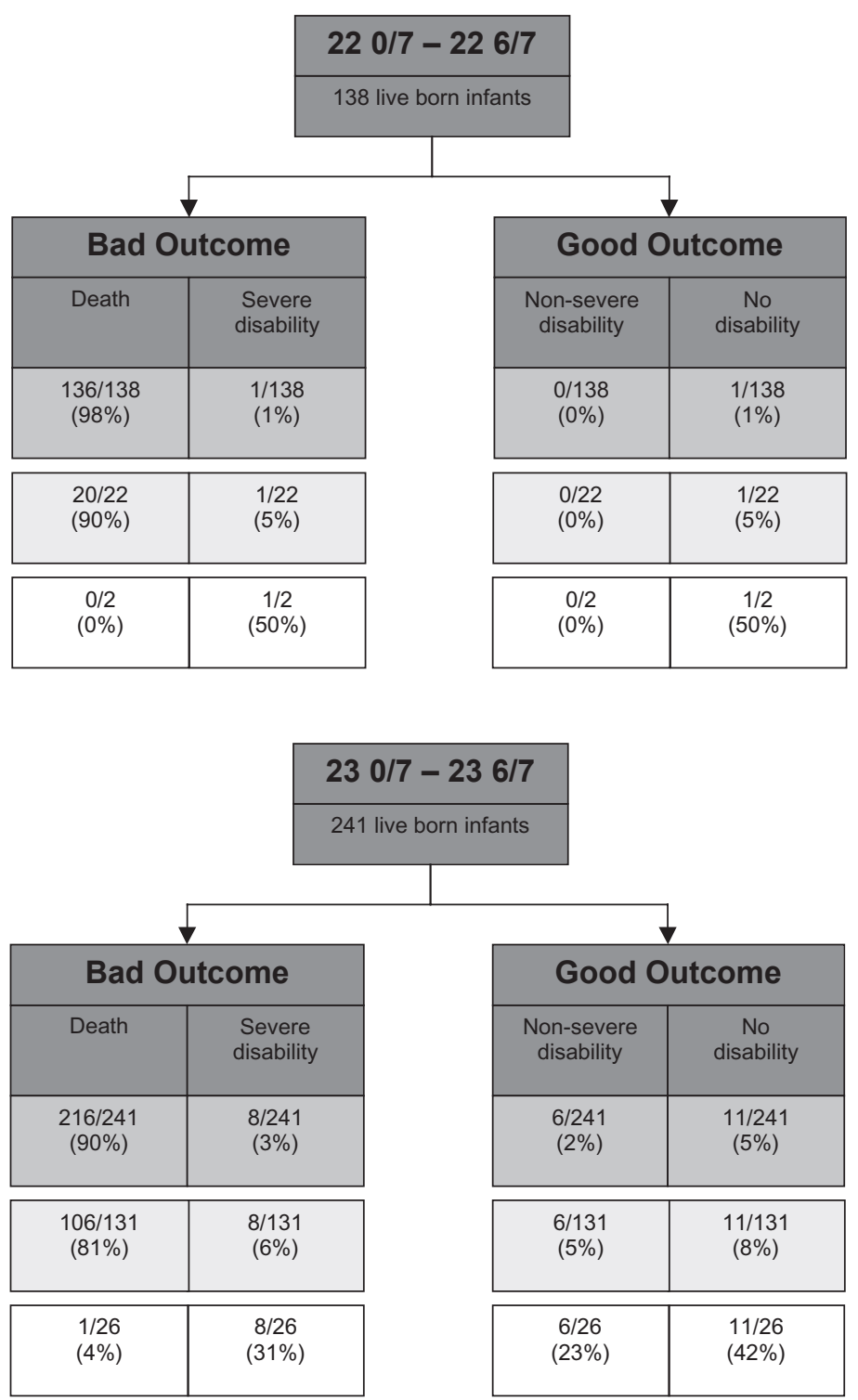

Si par principe, un jugement sur la qualité de vie humaine n'est pas permis, alors toute vie humaine doit être maintenue avec tous les moyens à disposition, entraînant un risque d'acharnement thérapeutique. D'autre part, si des thérapies prolongeant la vie ne sont acceptées qu'à condition d'obtenir une qualité de vie des plus élevée pour le prématuré traité, ceci pourrait être assimilé à une discrimination des personnes handicapées.

Un compromis possible entre ces deux positions éthiques extrêmes serait d'évaluer les thé- rapies envisagées de façon individuelle en comparant les résultats potentiels aux souffrances éventuelles liés à un traitement.

L'indication à renoncer ou à interrompre une thérapie chez un prématuré (voir 3.2.2) n'est pas motivée par le désir d'éviter une survie avec un handicap mais d'éviter des souffrances démesurées.

\subsubsection{Preneurs de décisions}

Puisque le prématuré dont la vie est directement touchée par les décisions thérapeutiques n'est pas en mesure d'exprimer ses préférences, les décisions doivent être par ses représentants. Par principe, les preneurs de décision sont soit l'équipe soignante, soit les parents de l'enfant ou la société sous forme d'une commission d'éthique ou d'un tribunal de justice.

Idéalement, les décisions ne sont pas prises ponctuellement par une seule instance mais élaborées dans un dialogue continu entre tous les participants (médecins, personnel soignant, parents). Une décision éthique est d'autant plus consistante que les parents ont été en mesure de la comprendre et de l'assumer, sans pour autant avoir le sentiment d'en porter seuls la responsabilité.

\subsubsection{Ressources du système de santé}

Lors de la prise en charge de prématurés extrêmes se pose la question si une proportion considérable de ressources financières disponibles peut être utilisée pour le traitement de prématurés à peine viables et à pronostic très défavorable alors que ces ressources pourraient manquer dans d'autres domaines du système de la santé.

Si une rationnement des moyens est nécessaire, il est plus juste d'exclure certaines thérapies spécifiques ayant un très mauvais rapport coût-bénéfice pour toutes les catégories de patients plutôt que d'exclure une catégorie spécifique de patients.

Par ailleurs, ces décisions devraient par principe être prise au niveau de la société de manière à ce que les facteurs économiques soient clairement séparés des pondérations éthiques pour un patient individuel.

\subsection{Communication}

Savoir communiquer un contenu complexe de manière adaptée à une situation donnée et aux partenaires présents demande beaucoup de compétence ainsi que beaucoup d'expérience. Il est ainsi crucial que de telles discussions soient conduites par des néonatologues expérimentés. Continuité et empathie jouent un rôle décisif, en particulier face à des parents. 
Tableau 2

Recommandations pour la prise en charge lors d'une menace d'accouchement prématuré avant 26 semaines révolues.

\begin{tabular}{|c|c|c|c|}
\hline $\begin{array}{l}\text { Age gestationel } \\
\text { (semaine de gestation révolue) }\end{array}$ & $\begin{array}{l}\text { Transport in utero } \\
\text { dans un centre périnatal }\end{array}$ & Césarienne & Prise en charge néonatale \\
\hline$<220 / 7$ & Non indiqué & Seulement sur indication maternelle & Comfort care \\
\hline $220 / 7-236 / 7$ & Eventuellement indiqué & Seulement sur indication maternelle & Comfort care \\
\hline $240 / 7-246 / 7$ & Indiqué & Rarement sur indication fœtale & $\begin{array}{l}\text { Mesures médicales intensives } \\
\text { selon situation individuelle* }\end{array}$ \\
\hline $250 / 7-256 / 7$ & Indiqué & Egalement sur indication fœtale & $\begin{array}{l}\text { Mesures médicales intensives } \\
\text { selon situation individuelle* }\end{array}$ \\
\hline
\end{tabular}

\subsubsection{Communication au sein de l'équipe de périnatologie}

La prise en charge d'un fœetus ou d'un prématuré à la limite de la viabilité doit être multidisciplinaire et implique une collaboration étroite entre obstétriciens, néonatologues, sages-femmes, personnel soignant et autres personnes impliquées. Souvent, les décisions doivent être prises en peu de temps. Il est donc nécessaire que les membres de l'équipe de périnatologie trouvent au préalable un consensus sur la manière de procéder lors de telles situations.

\subsubsection{Communication avec les parents}

La possibilité de prendre contact avec les futurs parents avant la naissance facilitera l'établissement d'une relation de confiance entre ces derniers et le néonatologue en vue de futures décisions éthiques communes. L'équipe soignante est tenue d'informer continuellement les parents sur les conséquences probables des décisions prises avec leur accord et qu'ils devront assumer par la suite. Les conseils aux parents doivent être francs et justes. Le temps à disposition pour assimiler l'information donnée doit être suffisant. Les parents ont souvent une représentation irréaliste non seulement des possibilités médicales mais aussi du pronostic de leur enfant, indépendamment du procédé thérapeutique proposé.

\section{Recommandations pour la prise en charge de prématurés à la limite de la viabilité}

\subsection{Avant la naissance}

\subsubsection{Transfert in utero}

Les critères pour un transfert in utero à temps doivent être clairement définis et régulièrement revus. Un transfert in utero dans un centre de pé- rinatologie doit être considéré dès la $22^{\mathrm{e}}$ semaine de gestation révolue. Même si aucune mesure médicale intensive ne sera prise en cas de naissance à cet âge (tabl. 2), le transfert de la parturiente permettra de mieux conseiller et préparer les parents (voir 3.1.2).

Ces derniers doivent être informés des buts du transfert in utero de façon à ce que leurs attentes soient adaptées à la situation et qu'elles restent réalistes.

\subsubsection{Information des parents}

Lorsque une naissance d'un prématuré à la limite de la viabilité s'annonce, le néonatologue responsable de la prise en charge de l'enfant devra être informé pour permettre une première discussion avec les parents.

Les parents doivent être informés des mesures médicales intensives probablement nécessaires durant les premiers jours de vie. Ils doivent également être renseignés sur les possibles complications. Lors de ce premier entretien avec les parents, il est important de percevoir leurs désirs et soucis sans pour autant s'attendre à des décisions claires. Cette attitude permet de réduire la pression et les sentiments de culpabilité parentaux.

Après une information approfondie et claire sur le pronostic probable de leur enfant, certains parents désireront l'abstention primaire des mesures de réanimation après la naissance. Bien que ce désir doit être respecté en général, et ceci surtout lors d'un âge gestationnel $<26$ semaines, il ne peut pas être imposé dans tous les cas au personnel soignant, ceci par exemple lors de la naissance d'un enfant s'avérant plus mature qu'estimé avant la naissance.

\subsubsection{Mesures obstétricales}

Lors de pronostic incertain pour l'enfant, les désirs des parents doivent êtres pris en considération lors de décisions obstétricales. Il n'existe que 


\section{Figure 1b}

Mortalité et morbidité neurologique et de prématurés d'âge gestationnel entre 24 0/7 et 25 6/7 (1995). Le développement psychomoteur a été évalué à l'âge de 30 mois (médian). Rectangles gris foncés: dénominateur = enfants nés vivants (live born infants), rectangles gris clairs: dénominateur = enfants admis en néonatologie (admissions to NICU), rectangles blancs: dénominateur = enfants vivants à la sortie d'hôpital (survivors to discharge). Donnés selon EPICure Study Group, 2000.
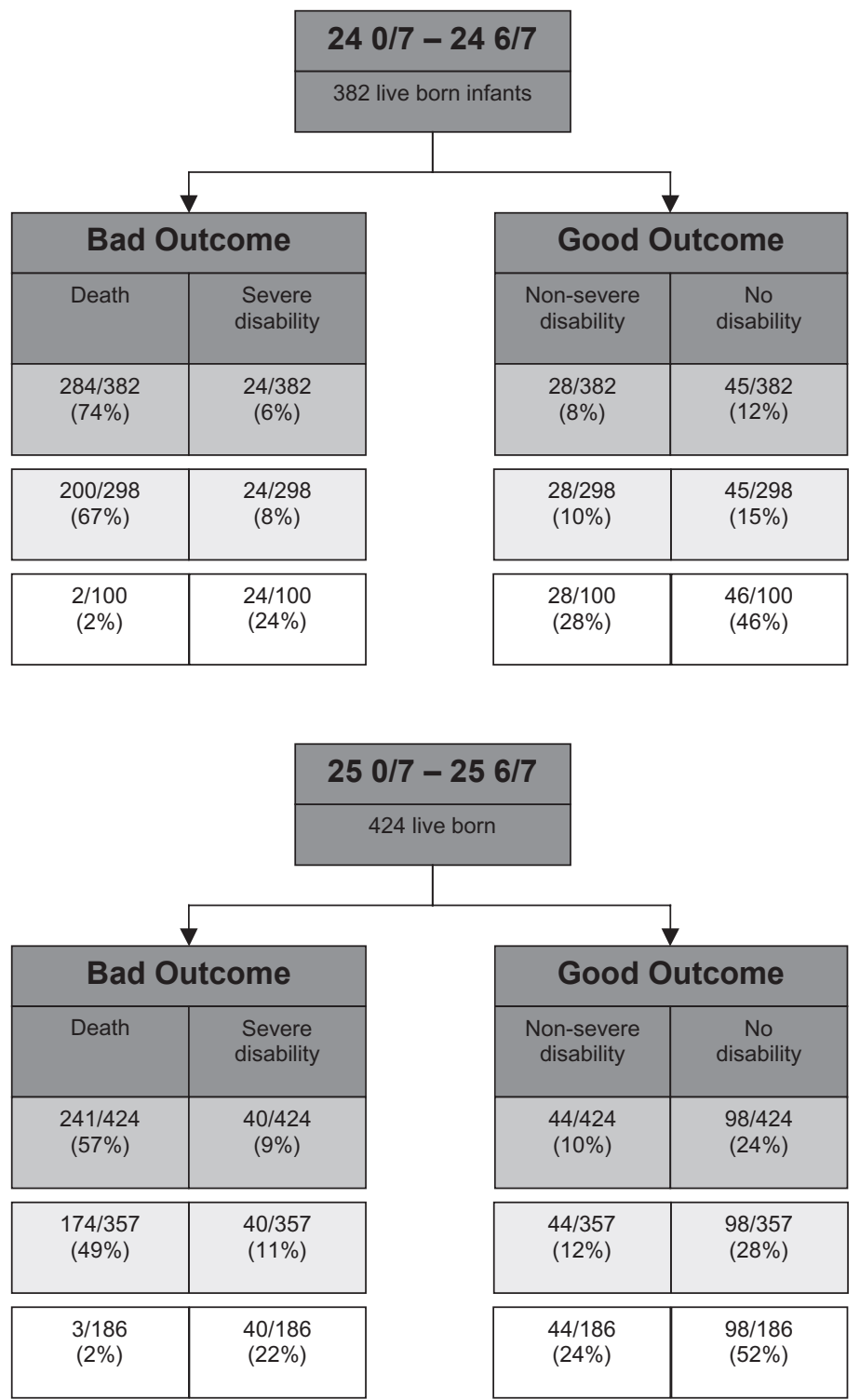

très peu d'études sur l'influence des interventions obstétricales, en particulier en ce qui concerne l'accouchement par césarienne sur les prématurés à la limite de la viabilité [13]. Les risques maternels sont à pondérer soigneusement avec les bénéfices potentiels pour le prématuré.

La naissance d'un prématuré à la limite de la viabilité doit évidemment être optimisée par les interventions obstétricales habituelles dans la mesure où les risques pour la mère, associés à ces manœuvres, restent acceptables.

\subsection{Après la naissance}

\subsubsection{Réanimation primaire en salle d'accouchement} La naissance d'un prématuré à la limite de la viabilité doit être considérée comme une urgence extraordinaire. Elle exige la présence d'obstétriciens et de néonatologues expérimentés afin d'être en mesure de prendre des décisions complexes quant à une réanimation intensive en salle d'accouchement, décisions difficiles et lourdes de conséquences.

La prise en charge individuelle sera influencée par des facteurs prénataux (âge gestationnel, poids de naissance, maturation pulmonaire par stéroïdes, retard de croissance intra-utérin, chorio-amnionite, malformations fotales, grossesse multiple), les désirs parentaux et l'état physique du prématuré immédiatement après la naissance (asphyxie, fréquence cardiaque, activité, réponse aux premières mesures de réanimation).

Les mesures obstétricales préalables (par exemple maturation pulmonaire, césarienne) ne doivent pas porter préjudice à l'initiation des traitements médicaux intensifs.

\subsubsection{Réanimation lors d'âge gestationnel} incertain. Une équipe de néonatologues doit être présente lors de chaque naissance à partir d'un âge $\geq 23$ semaines de gestation révolues afin de juger si des mesures intensives de réanimation sont justifiées par rapport à l'état physique du prématuré (voir 2.1).

Lors de situations incertaines, il peut être approprié d'initier une réanimation intensive puis de transférer le prématuré aux soins intensifs de néonatalogie (provisional intensive care), jusqu'à ce que l'évolution clinique et les discussions ultérieures avec les parents clarifient la question de la poursuite ou de l'arrêt des soins. Si la décision a été prise de faire bénéficier un prématuré du soi-disant provisional intensive care, cette prise en charge devra être optimale pour éviter tout dommage secondaire.

3.2.1.2 Réanimation lors d'âge gestationnel certain. La prise en charge de prématurés d'un âge gestationnel $<24$ semaines doit en général se limiter à des mesures palliatives (voir 3.2.2.3). Si par contre, un prématuré apparaît nettement plus mature à la naissance (voir 2.1) ou si après information préalable claire les parents insistent sur une prise en charge intensive, il est possible de commencer un traitement médical intensif dans le sens d'un provisional intensive care, jusqu'à ce que l'évolution ultérieure permette de décider si ces mesures médicales intensives doivent être poursuivies ou non. 
Lors de prise en charge de prématurés d'un âge gestationnel $\geq 24$ semaines, l'équipe néonatale doit décider en salle d'accouchement si la prise en charge médicale intensive est raisonnable. Une prise en charge médicale intensive provisoire permet souvent une évaluation détaillée de l'enfant dans un deuxième temps, de manière à mieux fonder la décision de continuer ou d'arrêter les mesures médicales intensives.

Le tableau 2 résume la prise en charge périnatale lors d'une menace d'accouchement avant 26 semaines d'âge de gestation révolues.

\subsubsection{Décisions ultérieures aux Soins Intensifs de Néonatologie}

3.2.2.1 Prise en charge intensive provisoire (provisional intensive care). La prise en charge médicale intensive débutée en salle d'accouchement et poursuivie aux soins intensifs de néonatologie est essentiellement guidée par un objectif thérapeutique. L'objectif premier est la survie de l'enfant avec une qualité de vie acceptable. Une limitation a priori des mesures médicales intensives basée uniquement sur l'âge gestationnel n'est pas justifiée dans cette situation précise. Les thérapies établies et acceptées en pratique clinique, dont on peut présumer qu'elles sont plus utiles que nuisibles, doivent être mises à disposition de chaque patient sans tenir compte de l'âge gestationnel.

Les mesures entreprises seront poursuivies aussi longtemps que les évaluations rapprochées permettent des espoirs fondés sur le but prédéfini et pour autant que les traitements nécessaires restent acceptables pour le prématuré. Les parents doivent être régulièrement informés de l'évolution.

3.2.2.2 Arrêt de soins intensifs (redirection of care). Si l'équipe soignante et les parents doivent reconnaitre que le but primaire ne peut pas être atteint, les mesures médicales intensives perdent leur sens et d'autres aspects de la prise en charge (par exemple les opiacés pour soulager douleurs et souffrances) deviennent prioritaires (redirection of care).

Les décisions d'un arrêt de la prise en charge médicale intensive doivent être soigneusement documentées dans le dossier du patient avec toutes les réflexions et raisons qui ont conduit à cette prise de position.

3.2.2.3 Prise en charge palliative (comfort care). Dès le moment où l'on renonce à mainte- nir artificiellement le prématuré en vie, tout doit être mis en œuvre pour offrir à l'enfant un décès digne (comfort care). Aussi faut-il faciliter les adieux des parents en favorisant un contact étroit avec leur enfant mourant.

Dans le but d'obtenir une analgésie efficace, on pourra administrer des doses d'opiacés pouvant éventuellement compromettre la survie [6]. L'administration par contre de médicaments avec l'intention de terminer la vie du prématuré est non seulement punissable mais aussi incompatible avec l'attitude éthique décrite sous le paragraphe 2.3.1.

\section{Recommandations pour la promotion et le contrôle de la qualité}

Le contrôle de qualité et sa promotion jouent un rôle primordial dans la prise en charge de prématurés à la limite de la viabilité. Incontestablement, les données de mortalité et morbidité de ce groupe de patients doivent être relevées de façon prospective et périodique, ceci aussi bien au niveau national que local. En plus des données périnatales déjà relevées au plan suisse dans le Minimal Neonatal DataSet (MNDS), on se doit de suivre également le développement psychomoteur et cognitif de ces enfants jusqu'à l'âge scolaire.

Puisque les décisions concernant les prématurés à la limite de la viabilité peuvent avoir des répercussions sur la famille entière [14], et que finalement seuls les patients et leurs parents peuvent juger de l'adéquation d'une prise en charge périnatale intensive, il est indispensable de récolter les données relatives au développement à long terme de ces enfants à la limite de la viabilité.

Une importante priorité doit être attribuée à la mise à disposition des moyens financiers pour la réalisation de telles études. En fonction des ces nouvelles données, ces présentes recommandations devront être réexaminées et modifiées si nécessaire.

\section{Remerciements}

Nous adressons notre gratitude à tous ceux qui ont d'une manière ou d'une autre contribué à ce document. Nous remercions Dr Riccardo Pfister et Mme Dr Cristina Exhery Pfister pour la traduction du texte en français. 


\section{Références}

1 American Academy of Pediatrics, Committee on Fetus and Newborn, and American College of Obstetricians and Gynecologists, Committee on Obstetric Practice. Perinatal care at the threshold of viability. Pediatrics 1995;96:974-6.

2 Canadian Paediatric Society, Fetus and Newborn Committee, and Society of Obstetricians and Gynaecologists of Canada, Maternal-Fetal Medicine Committee. Management of the woman with threatened birth of an infant of extremely low gestational age. CMAJ 1994;151:547-53.

3 Gee H, Dunn P, for the BAPM Executive Committee. Fetuses and newborn infants at the threshold of viability. A framework for practice, 2000.

4 Pohlandt F. Frühgeburt an der Grenze der Lebensfähigkeit des Kindes. PerinatalMedizin 1998;10:99-101.

5 SAMW. Medizinisch-ethische Richtlinien zu Grenzfragen der Intensivmedizin. Schweiz Ärztezeitung 1999;80:2134-8.

6 SAMW. Medizinisch-ethische Richtlinien für die ärztliche Betreuung sterbender und zerebral schwerst geschädigter Patienten. Schweiz Ärztezeitung 1995;76:1223-5.

7 Sabbagha R. Diagnostic ultrasound applied to obstetrics and gynecology. Gestational age. Philadelphia: Lippincott; 1987. p. 91-111.
8 Wood NS, Marlow N, Costeloe K, Gibson AT, Wilkinson AR. Neurologic and developmental disability after extremely preterm birth. EPICure Study Group. N Engl J Med 2000;343:378-84.

9 Jacobs SE, O’Brien K, Inwood S, Kelly EN, Whyte HE. Outcome of infants 23-26 weeks' gestation pre and post surfactant. Acta Paediatr 2000;89:959-65.

10 El-Metwally D, Vohr B, Tucker R. Survival and neonatal morbidity at the limits of viability in the mid 1990s: 22 to 25 weeks. J Pediatr 2000; 137:616-22.

11 Beauchamp T, Childress J. Principles of biomedical ethics. Oxford, New York, Toronto: Oxford University Press, 2001.

12 Kind C. Ethische Überlegungen als besondere Herausforderung für den Geburtshelfer und den Neonatologen. Der Gynäkologe 2001;34(8): 744-50.

13 Grant A. Elective versus selective caesarean section for delivery of the small baby. Cochrane Database Syst Rev 2000:CD000078.

14 Saigal S, Burrows E, Stoskopf BL, Rosenbaum PL, Streiner D. Impact of extreme prematurity on families of adolescent children. J Pediatr 2000; 137:701-6. 